\title{
HOPF bifurcation of the chemostat with delay and simplified holling type-iv response function
}

\section{Yanqiu $\mathrm{Li}^{*}$}

School of Applied Sciences, JiLin Teacher's Institute of Engineering And Technology, Changchun, China, 130052

Keywords: Chemostat, Holling type IV response, Delayed, Periodic solution.

\begin{abstract}
In this paper, the author investigates Chemostat with Delay and Simplifified Holling Type-IV Response Function, which more match the actual meaning in the chemostat system. Using bifurcation theory, we discuss the hopf bifurcation stability in detail.
\end{abstract}

\section{Introduction}

Chemostat is a amplified model of lakes, it has great significance in biology. On the Chemostat model of various types of equations, such as ordinary deferential equation, partial deferential equations and delay deferential equations. Standard microbial continuous culture model was developed in the period of 1940-1950.In the past 20 years, research about this model achieved abundant research results(see[1][2]). The single species model with time delay can be cultured with the following equation

$$
\left\{\begin{array}{c}
s^{\prime}(t)=\left(S_{0}-s(t)\right) D-\frac{1}{\delta} p(s(t)) x(t) \\
x^{\prime}(t)=-D x(t)+p(s(t-\tau)) x(t)
\end{array}\right.
$$

Here $s(t)$ is the concentration of nutrient at time $t, x(t)$ is the concentration of the microbial population at time $t ; D$ is rate of flow. Microbial consumption growth on nutrient medium rate is constant $1 / \delta ; \tau$ is the delay of time in converting nutrient into organism; $p(s)$ is the densitydependent uptake of nutrient by population

x. Holling(see[3]) first introduced the Holling II type functional response function

$$
p(s)=\frac{m s}{a+s}
$$

and Holling III type functional response function

\footnotetext{
*Corresponding author:343964060@qq.com
} 


$$
p(s)=\frac{m s^{2}}{a+s^{2}}
$$

which are monotonically increasing function.

There are a lot of results about the $\mathrm{p}(\mathrm{s})$ is a type of Holling II functional response.

$$
\left\{\begin{array}{c}
s^{\prime}(t)=1-s(t)-\frac{m s(t)}{a+s(t)} x(t) \\
x^{\prime}(t)=-x(t)+\frac{m s(t-\tau)}{a+s(t-\tau)} x(t)
\end{array}\right.
$$

Considering the system (4), the existence of internal equilibrium only $E^{*}=\left(s^{*}, a^{*}\right)=(a / m-$ $1, \mathrm{~m}-1-\mathrm{a} / \mathrm{m}-1)$ where $\mathrm{m}>\mathrm{a}+1$.

The hopf bifurcation existence and global periodic solution with the parameter $\tau$ about system (4) are discussed by reference [1], and the main conclusion is:

(1) if $0<\tau<\tau_{0}(\beta)$, then $E^{*}$ is asymptotically stable.

(2) if $\tau>\tau_{0}(\beta)$, then $E^{*}$ is not stable, and when

$$
\tau=\tau_{n}(\beta)=\frac{1}{k(\beta)}\left[\cos ^{-1} \frac{k^{2}(\beta)}{\beta}+2 n \pi\right], n=0,1,2 \ldots
$$

the system (4) produces hopf bifurcation, and there is one $\mathrm{T}>2 \tau$ non-constant periodic solution at least, where

$$
\beta=p^{\prime}(s) x^{*}, k(\beta)=\left[\frac{-(1+\beta)^{2}+\sqrt{(1+\beta)^{4}+4 \beta^{2}}}{2}\right]^{1 / 2}
$$

Considering the highly concentration medium on inhibition of microbial growth, Andrews[4] proposed Holling IV type functional response function which was non monotonic in 1968 ,

$$
p(s)=\frac{m s}{a+b s+s^{2}}
$$

Sokol and Howell proposed Holling IV type functional response function is simplified (see [5])

$$
p(s)=\frac{m s}{a+s^{2}}
$$

This function (6) is similar to function (5), and can better fit the experimental data (see [4], [6]), which contains only two parameters $\mathrm{m}$ and a, so the (5) is more widely used. This paper considers a simplified Holling IV type (6) system,

$$
\begin{gathered}
s=s_{0} \bar{s}, x=s_{0} \bar{x} \delta, t=\frac{\bar{t}}{D}, a=s_{0} \bar{a}, m=\bar{m} D, \tau=\frac{\bar{\tau}}{D}, t \in[-\tau, 0] \\
\left\{\begin{array}{l}
s^{\prime}(t)=1-s(t)-\frac{m s(t)}{a+s^{2}(t)} x(t) \\
x^{\prime}(t)=-x(t)+\frac{m s(t-\tau)}{a+s^{2}(t-\tau)} x(t)
\end{array}\right.
\end{gathered}
$$

where $\tau \geq 0, s(t)=\varphi(t) \geq 0, t \in[-\tau, 0], x(0)=x_{0}>0, \varphi$ is continuous when $t \in[-\tau, 0]$, $x(0)=x_{0}>0$.The system (7) can appear two internal equilibrium. With the author's 
knowledge, related literature give the static bifurcation analysis, given the region of internal equilibrium number division by the parameter, and gives the schematic diagram, determine the stability of the internal equilibrium about system (7), which is only the result of this equation. In the second section, we give a sufficient condition for the existence of a global periodic solutions of equation, by constructing specific function, analyzing the function of image structure, and use ejective point fix point theory to prove the existence of global periodic solution of this condition.

\section{HOPF bifurcation analysis}

Consider the equation:

$$
\left\{\begin{array}{c}
s^{\prime}(t)=1-s(t)-p(s(t)) x(t) \\
x^{\prime}(t)=-x(t)+p(s(t)) x(t)
\end{array}\right.
$$

The system of (8) is the following transformation: $\theta=\frac{t}{\tau}, \tilde{s}(\theta)=s(\tau \theta), \tilde{x}(\theta)=x(\tau \theta)$, and the $\mathrm{s}, \mathrm{X}$ instead of $\tilde{s}, \tilde{x},:\left\{\begin{array}{c}s^{\prime}(t)=\tau[1-s(t)-p(s(t)) x(t)] \\ x^{\prime}(t)=-\tau x(t)[-1+p(s(t))]\end{array}\right.$

Apparently if $\mathrm{p}(\mathrm{s})$ is $(6)$, we meet:

$$
\mathrm{p}(0)=0, \mathrm{p}(\mathrm{s})>0 \text {, for any } \mathrm{s}>0 \text {. }
$$

$$
\begin{gathered}
p^{\prime}(s)=\left\{\begin{array}{c}
>0,0 \leq s<\sqrt{a} \\
<0, s>\sqrt{a}
\end{array}\right. \\
p^{\prime \prime}(s)=\left\{\begin{array}{c}
>0,0 \leq s<\sqrt{3 a} \\
<0, s>\sqrt{3 a}
\end{array}\right. \\
\operatorname{pmax}(\mathrm{s})=\mathrm{p}(\sqrt{a})=\mathrm{m} / 2 \sqrt{a}
\end{gathered}
$$

It is obvious that no matter what parameter $m, a$ are, $\mathrm{E}_{0}=\left(\mathrm{s}_{0}, \mathrm{x}_{0}\right)=(1,0)$ is the system $(8)$ boundary equilibrium.If $\left(\mathrm{s}^{*}, \mathrm{x}^{*}\right)$ is a internal equilibrium, there must have the $\mathrm{p}\left(\mathrm{s}^{*}\right)=1, \mathrm{x}^{*}=1$ $s^{*}>0$..It is not difficult to prove the following conclusion:

Lemma 2.1. There is a constant $\mathrm{M}>0$, such that

$$
\max \left\{\lim _{t \rightarrow \infty} \sup s(t), \lim _{t \rightarrow \infty} \sup x(t)\right\} \leq M
$$

Lemma 2.2. There is a constant $\delta>0$, such that

$$
\lim _{t \rightarrow \infty} \text { inf } s(t) \geq \delta
$$

Lemma 2.3. $m<2 \sqrt{a}, a<1$, or $m=a+1, a \geq 1$, then

$$
\lim _{t \rightarrow+\infty} s(t)=1, \lim _{t \rightarrow+\infty} x(t)=0
$$

Lemma 2.4. If $m=2 \sqrt{a}, a<1$ or $m=a+1,0<a<1$ or $m>a+1$, the system of (8) has an internal equilibrium:

$$
E^{*}=\left(s^{*}, x^{*}\right)=\left(\frac{m-\sqrt{m^{2}-4 a}}{2}, 1-\frac{m-\sqrt{m^{2}-4 a}}{2}\right)
$$

Lemma 2.5. If $2 \sqrt{a}<m<a+1,0<a<1$, the system of (8) have two internal equilibrium:

$$
\begin{aligned}
& E_{1}=\left(s^{*}, x^{*}\right)=\left(\frac{m-\sqrt{m^{2}-4 a}}{2}, 1-\frac{m-\sqrt{m^{2}-4 a}}{2}\right) \\
& E_{2}=\left(s^{*}, x^{*}\right)=\left(\frac{m-\sqrt{m^{2}+4 a}}{2}, 1-\frac{m-\sqrt{m^{2}+4 a}}{2}\right)
\end{aligned}
$$

There is a concern of internal equilibrium, the direction of HOPF bifurcation and the stability of the nearby. In the case of $E_{1}, E_{2}$ in the same way. 
Translate $E_{1}$ to the origin:

$$
\left\{\begin{array}{l}
\dot{s}(t)=\tau\left[x^{*}-s(t)-p\left(s^{*}+s(t)\right)\left(x^{*}+x(t)\right)\right] \\
\dot{x}(t)=\tau\left(x^{*}+x(t)\right)\left[-1+p\left(s^{*}+s(t-1)\right)\right]
\end{array}\right.
$$

System (9) at the equilibrium point of $(0,0)$ the fifirst approximation system:

$$
\left\{\begin{array}{c}
\dot{s}(t)=-\tau(1+\beta) s(t)-\tau x(t) \\
\dot{x}(t)=\tau \beta s(t-1)
\end{array}\right.
$$

The characteristic equation of (10):

$$
\lambda^{2}+\tau(1+\beta) \lambda+\tau^{2} \beta e^{-\lambda}=0
$$

where $\beta=\dot{p}\left(s^{*}\right) x^{*} \geq 0$.

By reference,[1]: $\tau=\tau_{0}$, (11) has a simple pure imaginary root, and other roots have strictly negative real parts. Therefore, we use the methods in [7] and consider (9) about the bifurcation properties at $\tau=\tau_{0}$. According to the mathematical series, $\tau=\tau_{0}+\mu$ :

$$
\left\{\begin{array}{l}
\left.\dot{s}(t)=-\left(\tau_{0}+\mu\right)\left[(1+\beta) s(t)+x(t)+\frac{\beta}{x^{*}} x(t) s(t)+O(x(t)) s^{2}(t)\right)\right] \\
\left.\dot{x}(t)=\left(\tau_{0}+\mu\right)\left[\beta s(t-1)+\frac{\beta}{x^{*}} x(t) s(t-1)+O(x(t)) s^{2}(t-1)\right)\right]
\end{array}\right.
$$

The linear part:

$$
\left\{\begin{array}{c}
\dot{s}(t)=-\tau(1+\beta) s(t)-\tau x(t) \\
\dot{x}(t)=\tau \beta s(t-1)
\end{array}\right.
$$

The nonlinear part:

$$
\begin{gathered}
f\left(\mu, u_{t}\right)=\left(\begin{array}{c}
\left.-\left(\tau_{0}+\mu\right) \frac{\beta}{x^{*}} x(t) s(t)+O(x(t)) s^{2}(t)\right) \\
\left.\left(\tau_{0}+\mu\right) \frac{\beta}{x^{*}} x(t) s(t-1)+O(x(t)) s^{2}(t-1)\right)
\end{array}\right) \\
L_{\mu} \Phi=\left(\begin{array}{cc}
-\left(\tau_{0}+\mu\right)(1+\beta) & -\left(\tau_{0}+\mu\right) \\
0
\end{array}\right)\left(\begin{array}{l}
\varphi_{1}(0) \\
\varphi_{2}(0)
\end{array}\right)+\left(\begin{array}{cc}
0 & 0 \\
\left(\tau_{0}+\mu\right) \beta & 0
\end{array}\right)\left(\begin{array}{l}
\varphi_{1}(-1) \\
\varphi_{2}(-1)
\end{array}\right) \\
\eta(\theta, \mu)=\left(\begin{array}{cc}
-\left(\tau_{0}+\mu\right)(1+\beta) & -\left(\tau_{0}+\mu\right) \\
0
\end{array}\right) \delta(\theta)+\left(\begin{array}{cc}
0 & 0 \\
\left(\tau_{0}+\mu\right) \beta & 0
\end{array}\right) \delta(\theta+1) \\
0 \quad-1 \leq \theta<0 \\
R(\mu) \varphi=\left\{\begin{array}{c}
\frac{d \varphi(\theta)}{d \theta} \\
\int_{-1}^{0} d \eta(\theta, \mu) \Phi(\theta) \\
(0,0)^{T}
\end{array} \quad \begin{array}{c}
-1 \leq \theta<0 \\
\left(\begin{array}{c}
\left.-\left(\tau_{0}+\mu\right) \frac{\beta}{x^{*}} x(t) \varphi_{2}(0)+O\left(\varphi_{1}(0)\right) \varphi_{2}{ }^{2}(0)\right) \\
\left.\left(\tau_{0}+\mu\right) \frac{\beta}{x^{*}} \varphi_{1}(0) \varphi_{2}(-1)+O\left(\varphi_{1}(0)\right) \varphi_{2}{ }^{2}(-1)\right)
\end{array}\right)
\end{array} \quad \begin{array}{l}
\theta=0 \\
0
\end{array}\right.
\end{gathered}
$$

Therefore, the original formula is written by: 


$$
\dot{U}_{t}=A(\mu) U_{t}+R U_{t}
$$

where $U_{t}=(s(t), x(t))^{T}$.

$$
\begin{gathered}
\left(A^{*} \psi\right)(s)=\left\{\begin{array}{cc}
\frac{d \psi(s)}{d s} & 0<s \leq 1 \\
\int_{-1}^{0} d \eta^{T}(s, \mu) \Phi(s) & s=0
\end{array}\right. \\
(\psi, \varphi)=\bar{\psi}^{T}(0) \phi(0)-\int_{-1}^{0} \int_{0}^{\theta} \bar{\psi}^{T}(\xi-\theta)[d \eta(\theta)] \varphi(\xi) d \xi
\end{gathered}
$$

The $q(\theta)=\left(-\tau_{0}, i \omega_{0}+\tau_{0}(\beta+1)\right)^{T} e^{i \omega_{0} \theta}$ is a character vector operator about $\mathrm{A}$ on $i \omega_{0}$, $q^{*}(s)=N\left(-i \omega_{0},-\tau_{0}\right)^{T} e^{i \omega_{0} s}$ is a character vector operator about $\mathrm{A}$ on $-i \omega_{0}$, where $N=\left(-\tau_{0}^{2}(\beta+1)+2 \tau_{0} \omega_{0} i+\tau_{0}^{3} \beta e^{i \omega_{0}}\right)^{-1} . \quad z(t)=<q^{*}, u_{t}>, u_{t}=\left(u_{1 t}, u_{2 t}\right)$ is solution of (15) when $\mu=0$ note $\tau_{0}$ is $\tau, W(t, \theta)=\left(W^{1}(t, \theta), W^{2}(t, \theta)\right)^{T}=u_{t}-z(t) q(\theta)-\bar{z}(t) \bar{q}(\theta)$

where

$$
\dot{z}(t)=<q^{*}, u_{t}>=i \omega_{0} z(t)+\bar{q}^{*}(0) f_{0}(z, \bar{z})=i \omega_{0} z(t)+g(z, \bar{z})
$$

\section{And}

$$
g(z, \bar{z})=g_{20} \frac{z^{2}}{2}+g_{11} z \bar{z}+g_{02} \frac{\bar{z}^{2}}{2}+\cdots, \quad W\left(z \bar{z} \theta \neq W_{20} \theta\left(\frac{z^{2}}{2}\right)+W 1_{1} \theta \notin \bar{z}+W \frac{\bar{z}^{2}}{2^{2}}+\cdots\right.
$$

$$
f_{0}=\left(f_{0}^{1}, f_{0}^{2}\right)
$$

$f_{0}^{1}=-\frac{\tau^{3} \beta}{x^{*}}\left(N z^{2}+\bar{N} \bar{z}^{2}+(N+\bar{N}) z \bar{z}\right)+\frac{\tau^{2} \beta}{2 x^{*}}\left(W_{20}{ }^{1}(0) \bar{N}+W_{20}{ }^{2}(0)+2 W_{11}{ }^{1}(0) N+2 W_{11}{ }^{2}(0)\right) z^{2} z+\cdots$

$f_{0}^{2}=\frac{\tau^{3}}{x^{*}}\left(N e^{i \omega_{0}} z^{2}+\bar{N} e^{-i \omega_{0}} \bar{z}^{2}+\left(N e^{i \omega_{0}}+\bar{N} e^{-i \omega_{0}}\right) z \bar{z}\right)-\frac{\tau^{2}}{x^{*}}\left(W_{20}{ }^{1}(0) \frac{1}{2} \bar{N} e^{-i \omega_{0}}+W_{11}{ }^{1}(0) N e^{i \omega_{0}}+W_{11}{ }^{2}(-1)+\frac{1}{2} W_{20}{ }^{2}(-1)\right) z^{2} \bar{z}+\cdots$

$g(z, \bar{z})=\bar{q}^{* T}(0) f_{0}(z, \bar{z})=\bar{q}^{* 1}(0) f_{0}^{1}(z, \bar{z})+\bar{q}^{*}(0) f_{0}^{2}(z, \bar{z})$

$=-\frac{\tau^{3} N \bar{N}}{x^{*}}\left(\omega_{0} \beta i+\tau e^{i \omega_{0}}\right) z^{2}-\frac{\tau^{3} \bar{N}^{2}}{x^{*}}\left(\omega_{0} \beta i+\tau e^{-i \omega_{0}}\right) \bar{z}^{2}-\frac{\tau^{3} N}{x^{*}}\left((N+\bar{N}) \omega_{0} \beta i+\tau \bar{N} e^{-i \omega_{0}}+\tau N e^{i \omega_{0}}\right) z \bar{z}+\frac{\tau^{2} N}{2 x^{*}}$

$\left\{-\omega_{0} \beta i\left[W_{20}{ }^{1}(0) \bar{N}+W_{20}{ }^{2}(0)+2 W_{11}{ }^{1}(0) N+2 W_{11}{ }^{2}(0)\right]+\tau\left[\bar{N} W_{20}{ }^{1}(0) e^{-i \omega_{0}}+2 W_{11}{ }^{1}(0) N e^{i \omega_{0}}+2 W_{11}{ }^{2}(-1)+W_{20}{ }^{2}(-1)\right]\right\} z^{2} \bar{z}$.

Since

$$
\begin{aligned}
& \dot{W}=A W-2 \operatorname{Re}\left\{\bar{q}^{T}(0)\left(\begin{array}{c}
f_{0}^{1} \\
f_{0}^{2}
\end{array}\right) q(\theta)\right\}+\left\{\begin{array}{c}
(0,0)^{T}-1 \leq \theta<0 \\
\left(\begin{array}{l}
f_{0}^{1} \\
f_{0}^{2}
\end{array}\right) \quad \theta=0
\end{array}=A W+H(z, \bar{z}, \theta)\right. \\
& H(z, \bar{z}, \theta)=H_{20}(\theta) \frac{z^{2}}{2}+H_{11}(\theta) z \bar{z}+H_{02}(\theta) \frac{\bar{z}^{2}}{2}+\cdots W(z, \bar{z}, \theta)=W_{20}(\theta) \frac{z^{2}}{2}+W_{11}(\theta) z \bar{z}+W_{02} \frac{\bar{z}^{2}}{2}+\cdots
\end{aligned}
$$

Obtain

$$
\begin{gathered}
H_{20}(\theta)=\frac{2 \tau^{3} N \bar{N}}{x^{*}} a q(\theta)+\frac{2 \tau^{3} N^{2}}{x^{*}} \bar{b} \bar{q}(\theta) \\
H_{02}(\theta)=\frac{2 \tau^{3} \bar{N}^{2}}{x^{*}} b q(\theta)+\frac{2 \tau^{3} N \bar{N}}{x^{*}} \bar{b} \bar{q}(\theta) \quad H_{11}(\theta)=\frac{\tau^{3} \bar{N}}{x^{*}} c q(\theta)+\frac{\tau^{3} \bar{N}}{x^{*}} \bar{c} q(\theta) \\
a=\omega_{0} \beta i+\tau e^{i \omega_{0}}, b=\omega_{0} \beta i+\tau e^{-i \omega_{0}}, c=\omega_{0} \beta(N+\bar{N})+\tau\left(\bar{N} e^{-i \omega_{0}}+N e^{i \omega_{0}}\right) \\
H_{20}(0)=\frac{2 \tau^{3} N \bar{N}}{x^{*}} a q(0)+\frac{2 \tau^{3} N^{2}}{x^{*}} \bar{b} \bar{q}(0)+\left(\begin{array}{c}
-\frac{2 \tau^{3} N \beta}{x^{*}} \\
\frac{2 \tau^{3} N e^{i \omega_{0}}}{x^{*}}
\end{array}\right), \quad H_{11}(0)=\frac{\tau^{3} \bar{N}}{x^{*}} c q(0)+\frac{\tau^{3} \bar{N}}{x^{*}} \bar{c} q(0)+\left(\begin{array}{c}
-\frac{\tau^{3} \beta(N+\bar{N})}{x^{*}} \\
\frac{\tau^{3}\left(N e^{i \omega_{0}}+\bar{N} e^{-i \omega_{0}}\right)}{x^{*}}
\end{array}\right)
\end{gathered}
$$

According to [7] equation, $W_{11}$ and $W_{20}$ have the following results:

$\left(2 i \omega_{0}-A\right) W_{20}(\theta)=H_{20}(\theta), \quad-A W_{11}(\theta)=H_{11}(\theta)$

therefore

$$
W_{20}(\theta)=\left(\begin{array}{l}
W_{20}{ }^{1}(\theta) \\
W_{20}{ }^{2}(\theta)
\end{array}\right)=-\frac{2 \tau^{3} N \bar{N} i}{x^{*} \omega_{0}} a q(\theta)-\frac{2 \tau^{3} N^{2} i}{3 x^{*} \omega_{0}} b \bar{q}(\theta)+\left(\begin{array}{c}
E_{1} \\
E_{2}
\end{array}\right) e^{2 i \omega_{0} \theta} .
$$

Here 


$$
\begin{gathered}
E_{1}=-\frac{\tau^{3} N}{x^{*} \Delta}\left(4 \beta \omega_{0} i+2 \tau e^{i \omega_{0}}\right) \quad E_{2}=-\frac{2 \tau^{2} N \beta}{x^{*}}-\frac{E_{1}}{\tau}\left(2 \omega_{0} i+\tau(\beta+1)\right) \Delta=-4 \omega_{0}{ }^{2}+2 \omega_{0} \tau(\beta+1) i+\tau^{2} \beta e^{-2 i \omega_{0}} \\
W_{11}(\theta)=\left(\begin{array}{c}
W_{11}{ }^{1}(\theta) \\
W_{11}{ }^{2}(\theta)
\end{array}\right)=\frac{\tau^{3} \bar{N} i}{x^{*} \omega_{0}} c q(\theta)-\frac{\tau^{3} N i}{x^{*} \omega_{0}} \bar{c} \bar{q}(\theta)+\left(\begin{array}{c}
F_{1} \\
F_{2}
\end{array}\right)
\end{gathered}
$$

where

$$
F_{1}=-\frac{\tau^{2}}{x^{*} \beta}\left(N e^{i \omega_{0}}+\bar{N} e^{-i \omega_{0}}\right) \quad F_{2}=-\frac{\tau^{2}(1+\beta)}{x^{*} \beta}\left(N e^{i \omega_{0}}+\bar{N} e^{-i \omega_{0}}\right)-\frac{\tau^{2} \beta}{x^{*}}(N+\bar{N})
$$

The $W_{20}^{1}(0), W_{20}^{2}(0), W_{11}^{1}(0), W_{11}^{2}(-1), W_{20}^{2}(-1)$ substitution to $g_{21}$

$$
\begin{aligned}
g_{21} & =\frac{2 \tau^{5} \bar{N} i}{\omega_{0}\left(x^{*}\right)^{2}}\left\{\left(\tau(\beta+1)+i \omega_{0}-\tau N\right) N \bar{N} a^{2}+\tau N^{2} \bar{N}|a|^{2}+\left(\tau N+\frac{1}{3}\left(i \omega_{0}-\tau(\beta+1)\right)\right) a \bar{b}\right. \\
& +N \bar{N}\left(i \omega_{0}-\tau(\beta+1)\right) \bar{a} b+\left(\frac{1}{3} \tau \bar{N}+\left(\tau(\beta+1)-i \omega_{0}\right) N^{2} b \bar{b}+\left(\tau(\beta+1)+i \omega_{0}\right)(\bar{N}-N) a b\right\} \\
& +\frac{\tau^{2} N}{x^{*}}\left\{\bar{N} b E_{1}+2 N a F_{1}+\left(i \omega_{0} \beta+\tau e^{-2 i \omega_{0}}\right) E_{2}+2\left(i \omega_{0} \beta+\tau\right) F_{2}\right\} .
\end{aligned}
$$

Theorem 2.1. $\mu=0$ is a system of (12) Hopf bifurcation values, bifurcation direction is determined by the following formula: $\mu_{2}=-\operatorname{Re} C_{1}(0) / \alpha^{\prime}(0)$, The stability of bifurcating periodic solutions is determined by the following formula:

$\beta=2 \operatorname{Re} C_{1}(0), \quad$ where $C_{1}(0)=\frac{i}{2 \omega_{0}}\left[g_{20} g_{11}-2\left|g_{11}\right|^{2}-\frac{1}{3}\left|g_{02}\right|^{2}\right]+\frac{1}{2} g_{21}$

\section{Summary}

In this paper,the author investigates Chemostat with Delay and Simplifified Holling TypeIV Response Function, which more match the actual meaning in the chemostat system. Using bifurcation theory, we discuss the hopf bifurcation stability in detail.

This research was financially supported by the National Science Foundation (10571021).

\section{References}

1. Zhao Tao, Global periodic solutions for a diffferential delay system modeling a microbial population in the chemostat, Appl Math Anal,193 (1995) 329-352.

2. Ruan Shigui, Global analysis in a predator-prey system with nonmonotonic functional response ,SIAM J Appl Math,61(2001)1445-1472.

3. Holling $\mathrm{C} \mathrm{S}$, The functional response of predator to prey density and role in mimicry and population regulation, Men. Entomology. Soc. Can,45(1965)3-60.

4. Andrews J F, A mathematical model for the continuous culture of microorganisms utilizing inhibitory substrates, Biotechnol. Bioeng, 10(1968)707-723.

5. Sokol W and Howell J A, Kinetics of phenol oxidation by washed cells, Biotechnol. Bioeng, 23(1980)2039-2049.

6. Edwards $\mathrm{V} \mathrm{H}$, Inflfluence of high substrate concentrations on microbial kinetics, Biotechnol.Bioeng, 12(1970)679-712.

7. Hassard.B.D, Kazarinoffff.n.D. and Wan.Y.-H,Theory and Applications of Hopf Bifurcation, Cambridge University Press,London, 1981. 